\section{PDT-Metvix ${ }^{\circledR}$}

\author{
Galderma révèle l'étendue \\ de la uphase IV" de son \\ programme quant aux cancers \\ cutanés non mélanocytaires
}

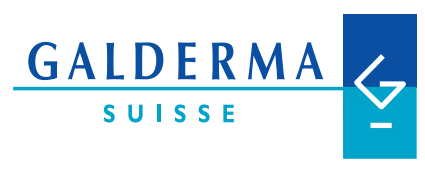

Metvix ${ }^{\circledR}$ est un nouveau traitement des cancers cutanés; il est absorbé par les cellules cancéreuses, puis activé après exposition à une lumière rouge spécifique (Aktilite ${ }^{\circledR}$ ). Metvix est recommandé pour le traitement des lésions pré-cancéreuses (kératoses actiniques, $\mathrm{AK}$ ) et les cancers cutanés non mélanocytaires (NMSC) dans la plupart des pays européens, la Nouvelle Zélande, l'Australie et est utilisé pour le traitement des AK aux Etats-Unis. Les autorisations de mise sur le marché dans de nombreux autres pays sont attendues prochainement.

À Florence, le 18 Novembre 2004, lors du $13 \mathrm{e}$ congrès de l'EADV, Galderma a annoncé les détails et l'ampleur de la $4 \mathrm{e}$ phase d'essais cliniques pour la thérapie photodynamique avec Metvix (PDT-Metvix) concernant plus de 800 patients atteints de NMSC dans 96 centres répartis dans le monde entier (Australie, Belgique, Allemagne, Italie, Espagne, Suisse et Grande-Bretagne).

Les détails de ce programme, composé de 8 études différentes s'ajoutent aux nombreuses informations déjà disponibles sur le Metvix. En terme de médecine factuelle («evidence-based medicine») et parmi les traitements des NMSC actuellement disponibles, celui par la PDT-Metvix présente la plus large évidence clinique comparative.

«C'est une série d'études très prometteuse» déclare le Dr Pascale Sota, directrice de la phase IV pour le laboratoire Galderma R\&D, Sophia Antipolis.

«Les NMSC, comme les carcinomes baso-cellulaires (BCC) et les AK sont des affections communes de la peau provo- quées par le soleil. Prises ensemble, ces maladies représentent un problème de santé publique grandissant pour des pays comme l'Australie, ceux d'Europe et les Etats-Unis où l'incidence est particulièrement élevée. Le programme étendu de la phase IV nous permettra d'obtenir l'image la plus claire jamais atteinte quant à l'efficacité du Metvix dans le traitement des AK et des BCC chez une grande quantité de patients.

Cette étude approfondira notre connaissance de l'efficacité de la PDTMetvix opposée aux méthodes habituelles de traitement des NMSC telles la chirurgie ou la cryothérapie. Bien que celles-ci soient efficaces, elles peuvent entraîner des cicatrices ou des dépigmentations, problèmes particulièrement sensibles pour les patients souffrant de lésions multiples au visage et autres localisations visibles.

Par contre, la PDT-Metvix est un traitement non invasif extrêmement sélectif. Très efficace pour soigner les $\mathrm{AK}$ et les BCC, il épargne la peau péri-lésionnelle saine donnant ainsi un résultat cosmétique très supérieur comparé aux autres thérapies invasives.»

L'efficacité de la PDT-Metvix est aussi étudiée chez des patients comme par exemple des transplantés ou des patients à haut risque.

Les premiers résultats concernant les patients transplantés seront disponibles dans le courant 2005.

Photocure SA est officiellement chargé de la publicité et de la distribution pour les pays nordiques, Galderma est responsable de la publicité et de la distribution de Metvix dans le monde entier.

\section{Nouveau \\ Flucazol $^{\circledR}$ l'antimycotique oral}

Avec l'introduction de Flucazol, on dispose désormais d'un générique du fluconazole avantageux. Il est possible d'économiser jusqu'à 46\% par rapport au produit original.

\section{Fluconazole - une substance connue, un schéma de posologie simple}

La substance active connue, le fluconazole, est utilisée lors de mycoses sévères et rebelles de la peau et des muqueuses lorsqu'un traitement topique ne suffit pas. La longue demi-vie ainsi que l'affinité élevée pour la peau permettent un schéma thérapeutique simple, p. ex.:

- candidose vaginale, $150 \mathrm{mg}$ dose unique;

- dermatomycoses (tinea pedis, tinea corporis, tinea cruris, infections à Candida), $150 \mathrm{mg}$ une fois par semaine ou $50 \mathrm{mg}$ par jour pendant 2-4 semaines.

\section{Une substance à la sécurité élevée}

Grâce aux différentes tailles et couleurs de comprimés, les diverses posologies peuvent être distinguées de manière optimale les unes des autres. Ainsi, toute confusion est pratiquement exclue. Chaque portion du blister est munie du nom du produit, de la substance, de la posologie et du nom de l'entreprise.

\section{Flucazol complète les produits topiques éprouvés que sont Imazol et Imacort}

Les topiques classiques se caractérisent par une galénique de pointe et une association de substances parfaitement adéquate:

Imazol crème-pâte, la préparation huile/eau hydrophile, absorbante avec adjonction de substance solide et $1 \%$ de clotrimazole est idéale lors de lésions suintantes. 
Imazol crème, la préparation hydrophile huile/eau avec $1 \%$ de clotrimazole et $0,25 \%$ d'hexamidine, est idéale lors d'infections mixtes.

Imacort crème, la préparation hydrophile huile/eau avec 1\% de clotrimazole, $0,25 \%$ d'hexamidine et $0,5 \%$ de prednisolone, est idéale lors d'infections mixtes très inflammatoires.

Grâce aux antimycotiques Spirig, vous disposez désormais d'un programme complet pour le traitement topique et systémique de pratiquement toutes les mycoses!

\section{Présentation}

\begin{tabular}{lll} 
& $\begin{array}{l}\text { Prix public } \\
\text { (y compris } \\
\text { TVA) }\end{array}$ & $\begin{array}{l}\text { Liste/liste } \\
\text { des } \\
\text { spécialités }\end{array}$ \\
\hline $\begin{array}{l}\text { Flucazol } 50 \mathrm{mg} \\
7 \text { capsules }\end{array}$ & 47,10 & $\begin{array}{l}\text { Liste B/LS dès } \\
1.11 .04\end{array}$ \\
\hline $\begin{array}{l}\text { Flucazol } 50 \mathrm{mg} \\
28 \text { capsules }\end{array}$ & 139,00 & $\begin{array}{l}\text { Liste } \mathrm{B} / \mathrm{LS} \text { dès } \\
1.11 .04\end{array}$ \\
\hline $\begin{array}{l}\text { Flucazol } 150 \mathrm{mg} \\
1 \text { capsule }\end{array}$ & 20,90 & $\begin{array}{l}\text { Liste B/LS dès } \\
1.11 .04\end{array}$ \\
\hline $\begin{array}{l}\text { Flucazol } 150 \mathrm{mg} \\
4 \text { capsules }\end{array}$ & 66,30 & $\begin{array}{l}\text { Liste B/LS dès } \\
1.11 .04\end{array}$ \\
\hline $\begin{array}{l}\text { Flucazol } 200 \mathrm{mg} \\
2 \text { capsules }\end{array}$ & 45,10 & $\begin{array}{l}\text { Liste B/LS dès } \\
1.11 .04\end{array}$ \\
\hline $\begin{array}{l}\text { Flucazol } 200 \mathrm{mg} \\
7 \text { capsules }\end{array}$ & 116,50 & $\begin{array}{l}\text { Liste B/LS dès } \\
1.11 .04\end{array}$ \\
\hline $\begin{array}{l}\text { Imazol } \\
\text { crème-pâte } 30 \mathrm{~g}\end{array}$ & 15,75 & Liste C/LS \\
\hline \begin{tabular}{l} 
Imazol crème $30 \mathrm{~g}$ \\
\hline Imacort crème $20 \mathrm{~g}$
\end{tabular} & 17,85 & Liste B/LS \\
\hline Imas & Liste B/LS \\
\hline
\end{tabular}

Pour de plus amples informations, veuillez contacter:

Sibylle Waldmeier

Product Manager

Spirig Pharma AG

Froschackerstrasse 6

$\mathrm{CH}-4622$ Egerkingen

Tél. 0623878787

E-mail sibylle.waldmeier@spirig.ch

Flucazol ${ }^{\circledR}$. C: fluconazole; capsules à $50 \mathrm{mg}, 150 \mathrm{mg}, 200 \mathrm{mg}$. I: candidose des muqueuses, y compris prophylaxie chez les patients présentant une neutropénie induite par la chimiothérapie ou la radiothérapie, candidémie, dermatomycoses, méningite à cryptocoques chez les patients atteints de SIDA. CI: traitement simultané par terfénadine, cisapride; grossesse (jusqu'à 7 jours après le traitement), allaitement. EI: troubles gastro-intestinaux, éruptions cutanées, valeurs anormales des tests de la fonction rénale et hépatique (en particulier chez les patients présentant des formes graves de maladie de base). IA: substances métabolisées par les isoenzymes CYP 2C9, CYP 2D6 ou CYP 3A4. P: candidose oropharyngée: $50(-100) \mathrm{mg} /$ jour pendant 7-14 jours; candidose œsophagienne: $400 \mathrm{mg}$ en dose unique; candidose vaginale: $150 \mathrm{mg}$ en dose unique; autres candidoses: $50 \mathrm{mg}$ /jour pendant 14-30 jours. Liste B, liste des spécialités.

Pour de plus amples informations, consultez le Compendium suisse des médicaments.

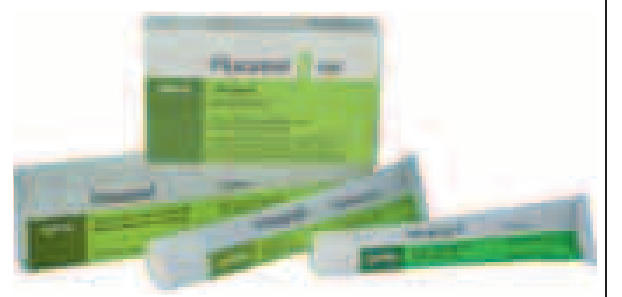

\section{Flucazol ergänzt die bewährten Topika Imazol und} Imacort

Die klassischen Topika zeichnen sich durch pfiffige Galenik und optimal aufeinander abgestimmte Wirkstoffkombinationen aus:

Imazol Cremepaste, die hydrophile, saugfähige Öl-in-Wasser-Zubereitung mit Feststoffzusatz und 1\% Clotrimazol, ist ideal bei nässenden Läsionen.

Imazol Creme, die hydrophile Öl-inWasser-Zubereitung mit 1\% Clotrimazol und $0,25 \%$ Hexamidin, ist ideal bei Mischinfektionen.

\section{Neu \\ Flucazol $^{\circledR}$ - orales $^{2}$ Antimykotikum}

Mit der Einführung vom Flucazol steht neu ein kostengünstiges Fluconazol-Generikum zur Verfügung. Gegenüber dem Originalpräparat können bis $46 \%$ eingespart werden.

\section{Fluconazol - bekannter Wirkstoff, einfaches Dosierungsschema}

Der bekannte Wirkstoff Fluconazol wird bei schwereren und hartnäckigen Mykosen der Haut und der Schleimhäute eingesetzt, wenn eine topische Behandlung nicht ausreichend ist. Die lange Halbwertszeit und die hohe Hautaffinität ermöglichen einfache Therapieschemata, z. B.:

- vaginale Candidose, 150 mg Einmaldosis;

- Dermatomykosen (Tinea pedis, Tinea corporis, Tinea cruris, Candida-Infektionen), $150 \mathrm{mg}$ einmal wöchentlich oder 50 mg täglich während 2-4 Wochen.
Imacort Creme, die hydrophile Ölin-Wasser-Zubereitung mit 1\% Clotrimazol, 0,25\% Hexamidin und 0,5\% Prednisolon, ist ideal bei stark entzündlichen Mischinfektionen.

Mit dem Spirig-Antimykotika-Set steht Ihnen jetzt ein komplettes Programm zur topischen und systemischen Behandlung fast aller Mykosen zur Verfügung!

\section{Präsentation}

\begin{tabular}{lll} 
& $\begin{array}{l}\text { Publikums- } \\
\text { preis }\end{array}$ & $\begin{array}{l}\text { Liste/Spezia- } \\
\text { litätenliste }\end{array}$ \\
\hline $\begin{array}{l}\text { Flucazol } 50 \mathrm{mg} \\
7 \text { Kapseln }\end{array}$ & 47.10 & $\begin{array}{l}\text { Liste B/SL ab } \\
1.11 .04\end{array}$ \\
\hline $\begin{array}{l}\text { Flucazol } 50 \mathrm{mg} \\
28 \text { Kapseln }\end{array}$ & 139.00 & $\begin{array}{l}\text { Liste B/SL ab } \\
1.11 .04\end{array}$ \\
\hline $\begin{array}{l}\text { Flucazol } 150 \mathrm{mg} \\
1 \text { Kapsel }\end{array}$ & 20.90 & $\begin{array}{l}\text { Liste B/SL ab } \\
1.11 .04\end{array}$ \\
\hline $\begin{array}{l}\text { Flucazol } 150 \mathrm{mg} \\
4 \text { Kapseln }\end{array}$ & 66.30 & $\begin{array}{l}\text { Liste B/SL ab } \\
1.11 .04\end{array}$ \\
\hline $\begin{array}{l}\text { Flucazol } 200 \mathrm{mg} \\
2 \text { Kapseln }\end{array}$ & 45.10 & $\begin{array}{l}\text { Liste B/SL ab } \\
1.11 .04\end{array}$ \\
\hline $\begin{array}{l}\text { Flucazol } 200 \mathrm{mg} \\
7 \text { Kapseln }\end{array}$ & 116.50 & $\begin{array}{l}\text { Liste B/SL ab } \\
1.11 .04\end{array}$ \\
\hline $\begin{array}{l}\text { Imazol } \\
\text { Cremepaste } 30 \mathrm{~g}\end{array}$ & 15.75 & Liste C/SL \\
\hline Imazol Creme $30 \mathrm{~g}$ & 17.85 & Liste B/SL \\
\hline Imacort Creme $20 \mathrm{~g}$ & 16.95 & Liste B/SL \\
\hline
\end{tabular}


Weitere Informationen erhalten Sie bei:

Sibylle Waldmeier

Product Manager

Spirig Pharma AG

Froschackerstrasse 6

CH-4622 Egerkingen

Tel. 0623878787

E-Mail sibylle.waldmeier@spirig.ch

Flucazol $^{\circledR}$. Z: Fluconazol; Kapseln $50 \mathrm{mg}, 150 \mathrm{mg}, 200 \mathrm{mg}$. I: SchleimhautCandidosen, Candidämie, Dermatomykosen, Kryptokokkenmeningitis bei AIDS-Patienten. KI: Gleichzeitig mit Terfenadin, Cisaprid; Schwangerschaft (bis 7 Tage nach Therapie), Stillzeit. NW: gastrointestinale Störungen, Hautausschläge, pathologische Nieren- und Leberwerte sowie hämatologische Störungen (insbesondere bei Patienten mit schweren Grundkrankheiten). IA: Substanzen, die über die Isoenzyme CYP 2C9, CYP 2D6 oder CYP 3A4 metabolisiert werden. D: oropharyngeale Candidose: 50(-100) mg täglich während 7-14 Tagen; ösophageale Candidose: $400 \mathrm{mg}$ als Einmaldosis; vaginale Candidose: $150 \mathrm{mg}$ als Einmaldosis; andere Candidosen: 50 mg täglich während 1430 Tagen. Liste B, Spezialitätenliste.

Weitere Informationen im «Arzneimittelkompendium der Schweiz».

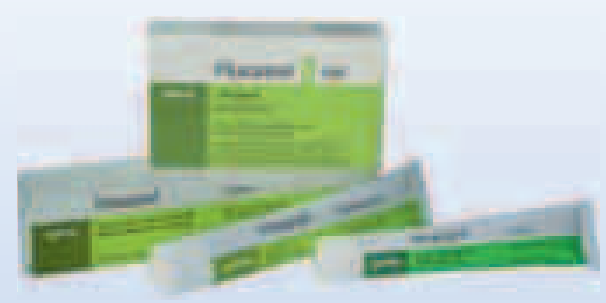

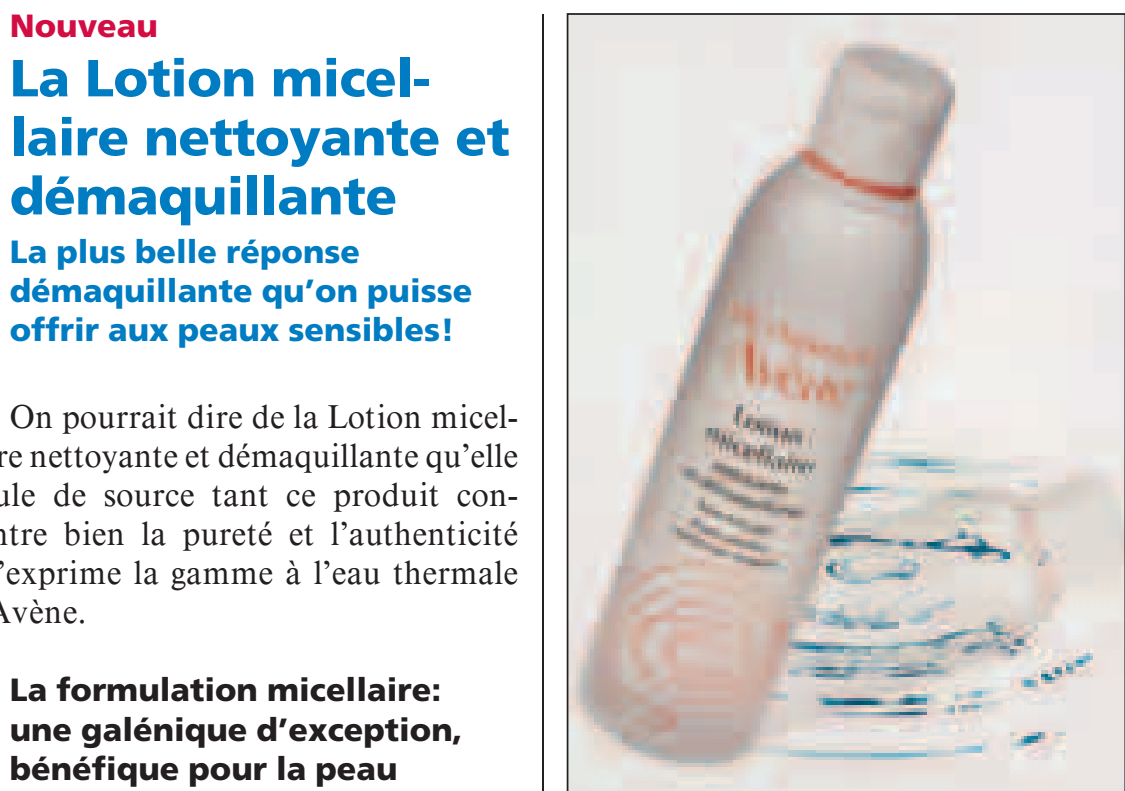

Un formulateur, tout comme un grand chef, a l'art de marier les constituants pour en obtenir le meilleur.

Ainsi, il sait qu'à une certaine concentration, les tensio-actifs (constitués d'une tête hydrophile et d'un corps hydrophobe) sont capables de s'organiser en «micelles». En solution aqueuse, ces micelles ressemblent à des sphères dont la partie externe est constituée des «têtes hydrophiles» et la partie interne des «corps hydrophobes». Dans leur partie interne peuvent se solubiliser des principes actifs non solubles dans l'eau.

Une lotion qui contient des tensioactifs organisés de la sorte est dite «micellaire». Il s'agit d'une lotion homogène, monophasique (constituée d'une seule phase) et dont les principes actifs sont dissous. Ces principes actifs seront libérés par le contact avec la peau.

Pour tout renseignement complémentaire veuillez contacter:

Laboratoires dermatologiques Avène

infomed@avene.ch
Neu

\section{Nanolotion zur} Gesichtsreinigung und Make-upEntfernung

Ultramilde Reinigungspflege speziell für die empfindliche Haut

Die Nanolotion zur Gesichtsreinigung und Make-up-Entfernung harmoniert mehr denn je mit dem Begriff von Reinheit und Authentizität der Pflegelinie mit Avène-Thermalwasser.

\section{Eine in Form von Mizellen angeordnete Formel: aussergewöhnliche Galenik, wohltuende Wirkung auf die Haut}

Die besondere Fähigkeit einer chemischen Formel besteht darin, Ingredienzien harmonisch aufeinander abzustimmen, um ein optimales Ergebnis zu erhalten. Wasser abweisenden (hydrophoben) und Wasser anziehenden (hydrophilen) Teilen bestehen. Ab einer bestimmten Konzentration besitzen sie die Fähigkeit, sich in Form von Mizellen spontan zusammenzulagern. In einer wässrigen
Heute weiss man, dass Tenside aus 
Lösung können diese Mizellen mit einer Sphäre verglichen werden, deren äusserer Teil aus einem «hydrophilen Kopf» und deren innerer Teil aus einem «hydrophoben Schwanz» besteht. Im inneren Teil der Mizelle können sich die zuvor wasserunlöslichen Aktivstoffe wieder im Wasser auflösen.

Unter dem Begriff «Nanotechnologie» versteht man, unter anderem, eine Lotion, in der sich Tenside in oben erwähnter Art und Weise anordnen. Es handelt sich dabei um eine homogene Lotion, in der sich die Aktivstoffe zusammenlagern, um dann eine eigene Phase zu bilden, die beim Kontakt mit der Haut freigesetzt wird.

Für weitere Auskünfte wenden Sie sich bitte an: Laboratoires dermatologiques Avène infomed@avene.ch

\section{Raptiva ${ }^{\circledR}$,}

premier traitement biologique du psoriasis en plaques, à être admis aux caisses-maladie

L'office fédéral de la santé publique a inscrit Raptiva ${ }^{\circledR}$ (éfalizumab) sur la liste de spécialités à compter du 1er janvier 2005. Raptiva devient ainsi le premier traitement biologique du psoriasis en plaques à être remboursé par l'assurance de base. Son remboursement est soumis à la condition suivante:

Traitement des patients adultes atteints des formes sévères de psoriasis en plaques qui n'ont pas répondu à la photothérapie UVB et à la PUVAthérapie ou à l'un des trois traitements systémiques (ciclosporine, méthotrexate, acitrétine). En l'absence de succès thérapeutique après 12 semaines, le traitement doit être interrompu. L'ordonnance ne peut être rédigée que par des dermatologues ou des hôpitaux universitaires/policliniques spécialisé(e)s en la dermatologie.

\section{Prescription de Raptiva}

Le dermatologue peut faire une ordonnance pour Raptiva ou une prescription par la SVK (Fédération suisse pour tâches communes des assureurs-maladie) offrant les avantages suivants:

- Uniforme et rapide pour toutes les caisses-maladie affiliées à la SVK

- Exclusivement réservée aux dermatologues

- Facturation directe à la caissemaladie

- Après concertation avec la SVK, les coûts des médicaments réglés par la SVK ne sont pas pris en considération dans la statistique du centre de calcul de Santésuisse.

Raptiva est disponible en boîtes de 4 ampoules.

Prix ex factory: CHF 1426,90

(TVA excl.)

Prix public: CHF 1668,70

(TVA incl.)

Serono Pharma Suisse

Steinhauserstrasse 74

CH-6305 Zoug

Tél. 0417480060

Fax 0417480070

E-mailinfo@raptiva.ch

\section{Raptiva ${ }^{\circledR}$ erstes Biologikum zur Therapie der Plaquepsoriasis, kassenpflichtig}

Das Bundesamt für Gesundheit hat per 1. Januar 2005 Raptiva ${ }^{\circledR}$ (Efalizu$\mathrm{mab}$ ) in die Spezialitätenliste aufgenommen. Damit ist Raptiva das erste Biologikum zur Therapie der Plaquepsoriasis, welches aus der Grundversicherung vergütet wird. Die entsprechende Limitatio lautet:

Behandlung erwachsener Patienten mit schwerer Plaquepsoriasis, bei denen UVB und PUVA oder eine der drei systemischen Therapien (Ciclosporin, Methotrexat, Acitretin) keinen therapeutischen Erfolg gezeigt haben. Falls nach 12 Wochen kein therapeutischer Erfolg eingetreten ist, ist die Behandlung abzubrechen. Die Verschreibung kann nur durch Fachärzte der Dermatologie oder dermatologische Universitätskliniken/Polikliniken erfolgen.

\section{Verschreibung von Raptiva}

Neben der klassischen Verschreibung via Rezept besteht für Dermatologen die Möglichkeit, die Verordnung von Raptiva via SVK (Schweizerischer Verband für Gemeinschaftsaufgaben der Krankenversicherer) vorzunehmen. Dies bietet folgenden Nutzen:

- Einheitlich und rasch für alle dem

SVK angeschlossenen Krankenkassen

- Exklusiv für Dermatologen

- Direkte Fakturierung an die Krankenkasse

- Nach Rücksprache mit dem SVK gilt, dass Medikamentenkosten, die via SVK abgerechnet werden, in der Rechnungssteller-Statistik der Santésuisse nicht berücksichtigt werden.

Raptiva ist in Packungen zu 4 Durchstechflaschen erhältlich: Fabrikabgabepreis: CHF 1426.90 (exkl. MWSt)

Publikumspreis: CHF 1668.70 (inkl. MWSt.)

Serono Pharma Schweiz

Steinhauserstrasse 74

CH-6305 Zug

Tel. 0417480060

Fax 0417480070

E-Mail info@raptiva.ch 